\title{
Model based AHP
}

\author{
Kazuyuki Sekitani* and Naokazu Yamaki \\ Department of Systems Engineering, Shizuoka University \\ 3-5-1 Jyouhoku, Hamamatsu, Shizuoka 432-8561 Japan \\ sekitani@sys.eng.shizuoka.ac.jp
}

\begin{abstract}
Sekitani and Yamaki introduced two new concepts of self-evaluation value and non-self-evaluation value into $\mathrm{AHP}$ and showed that the eigenvalue method can be formulated as some mathematical programming problems with the ratios of the self-evaluation value to the non-self-evaluation value. This study develops a new discrepancy-minimization problem with the ratios of the self-evaluation value to the non-self-evaluation value and their reciprocals. We show that its optimal solution is identical to the principal eigenvectors of the pairwise comparison matrix. We compare the analytical properties of the proposed optimization model with that of Harker method for the case of AHP with incomplete information.
\end{abstract}

\section{Introduction}

The eigenvalue method (EM), that is to find a principal eigenvector of a pairwise comparison matrix, is widely used in AHP. It has been suggested that Frobenius' theorems play an important role to guarantee the existence and the uniqueness of the weight vector which is provided by EM.

Recently Sekitani and Yamaki (Sekitani and Yamaki,1999) focused on Frobenius' min-max theorem from the viewpoint of mathematical programming and then introduced two new concepts of selfevaluation value and non-self-evaluation value into AHP. The $i^{\text {th }}$ self-evaluation value is the $i^{\text {th }}$ component $w_{i}$ of the weight vector $w$ and the $i^{t h}$ non-self-evaluation value is $\left(a_{i} w-w_{i}\right) /(n-1)$, where $a_{i}$ is the $i^{\text {th }}$ row vector of the pairwise comparison matrix of order $n$. They showed that Frobenius' min-max theorem is interpreted as the following two optimization models $P_{1}$ and $P_{2}$ :

$$
\begin{aligned}
& \left(P_{1}\right) \quad \max _{w>0} \min \left\{\frac{a_{1} w-w_{1}}{(n-1) w_{1}}, \ldots, \frac{a_{n} w-w_{n}}{(n-1) w_{n}}\right\} \\
& \left(P_{2}\right) \quad \min _{w>0} \max \left\{\frac{a_{1} w-w_{1}}{(n-1) w_{1}}, \ldots, \frac{a_{n} w-w_{n}}{(n-1) w_{n}}\right\}
\end{aligned}
$$

The model $P_{1}$ minimizes the largest ratio of $w_{i}$ to $\left(a_{i} w-w_{i}\right) /(n-1)$ and the model $P_{2}$ maximizes the least ratio of $w_{i}$ to $\left(a_{i} w-w_{i}\right) /(n-1)$. Sekitani and Yamaki also proved that the optimal solutions of both $P_{1}$ and $P_{2}$ are identical and equivalent to a principal eigenvector of the pairwise comparison matrix.

In this study, in order to combine the two optimization models $P_{1}$ and $P_{2}$, we develop a new discrepancy-minimization problem that evaluates the ratios of $w_{i}$ to $\left(a_{i} w-w_{i}\right) /(n-1)$ and their reciprocals.

AHP assumes that all pairs of alternatives/objects should be compared. Therefore, AHP with incomplete pairwise comparisons is an exceptional case that needs a special method, e.g., Harker method (Harker,1987). In this study, we apply the above three models $\left(P_{1}, P_{2}\right.$ and the combined one) to the case of AHP with incomplete information as a natural extension of the models. We show that these three models can deal with AHP with incomplete pairwise comparisons as well as that with all pairwise comparisons.

From the graph-theoretical argument we discuss the analytical properties of these three optimization models through the comparisons with Harker method.

\footnotetext{
"The author was partially supported by Grand-in-Aid for Scientific Research of the Ministry of Education, Science, Sports and Culture, Grant No. 11780328 and Shizuoka University fund for engineering research.
} 


\section{Fundamental Theorems and Generalization of Key Concepts}

AHP with all pairwise comparisons provides the pairwise comparison matrix $A$ whose $(i, j)$ entry is the pairwise comparison value $a_{i j}$ of the $i^{t h}$ alternative/object and the $j^{t h}$ alternative/object. Because the pairwise comparison value $a_{i j}$ is defined positive, the comparison matrix $A$ is positive and irreducible. Therefore the following well-known theorem guarantees that the weight vector by EM is unique, and that it is positive.

Theorem 1 (Perron-Frobenius' Theorem (Takayama,1985)) Suppose that $A$ is an irreducible nonnegative matrix. Then there are an eigenvalue $\lambda$ and the corresponding eigenvector $w$ satisfying the following two conditions:

(1) $A w=\lambda w, \lambda>0, w>0$ and $\lambda \geq|\alpha|$ for every eigenvalue $\alpha$ of the matrix $A$.

(2) $\lambda$ is a single root of the characteristic equation of $A$.

Furthermore Frobenius' min-max theorem states that the two mathematical programming problems (stated below) have the same optimal solution and that it is identical to the weight vector of EM.

Theorem 2 (Frobenius' min-max Theorem (Furuya,1957)) Suppose that $A$ is a nonnegative matrix of order $n$, and that $\lambda_{\max }$ is the principal eigenvalue of $A$. Then for every $n$-dimensional positive vector $w$,

$$
\min \left\{\frac{a_{1} w}{w_{1}}, \ldots, \frac{a_{n} w}{w_{n}}\right\} \leq \lambda_{\max } \leq \max \left\{\frac{a_{1} w}{w_{1}}, \ldots, \frac{a_{n} w}{w_{n}}\right\}
$$

Furthermore, if the matrix $A$ is irreducible,

$$
\max _{w>0} \min \left\{\frac{a_{1} w}{w_{1}}, \ldots, \frac{a_{n} w}{w_{n}}\right\}=\lambda_{\max }=\min _{w>0} \max \left\{\frac{a_{1} w}{w_{1}}, \ldots, \frac{a_{n} w}{w_{n}}\right\},
$$

where the two equalities in (2) hold for every positive eigenvector $w$ corresponding to the principal eigenvalue $\lambda_{\max }$.

Sekitani and Yamaki proposed the model-based AHP with all pairwise comparisons of $n$ alternatives as follows: the model-based AHP supposes that every alternative evaluates itself, and that it gives itself a positive real number. Let $w_{i}$ be the positive real number given to the $i^{\text {th }}$ alternative by itself. The value $w_{i}$ of the $i^{\text {th }}$ alternative is called the $i^{\text {th }}$ self-evaluation value. The value $a_{i j} w_{j}$ represents the evaluation value of the $i^{\text {th }}$ alternative from the viewpoint of the $j^{\text {th }}$ alternative when the $j^{\text {th }}$ self-evaluation value is $w_{j}$. It is called external evaluation of $i$ by Takahashi (Takahashi,1999). Since the number of the alternatives is $n$, the number of the evaluation values of the $i^{t h}$ alternative from the viewpoint of others is $n-1$. Averaging $a_{i j} w_{j}$ over $j$ except $i$, we obtain $\left(\sum_{j \neq i} a_{i j} w_{j}\right) /(n-1)$ This we can call the "averaging principle." Because $a_{i i}=1$, we call $\left(a_{i} w-w_{i}\right) /(n-1)$ the $i^{t h}$ non-self-evaluation value.

For AHP with the incomplete pairwise comparisons, let $a_{i j}$ be the pairwise comparison value when the pair of the alternatives $i$ and $j$ is evaluated by a decision maker, and let $a_{i j}$ be 0 when the pair of the alternatives $i$ and $j$ is not evaluated. Let $a_{i i}=1$ for $i=1, \ldots, n$ and $a_{j i}=1 / a_{i j}$ for $a_{i j}>0$. Then the nonnegative matrix $A=\left(a_{i j}\right)$ is well defined. We call $A=\left(a_{i j}\right)$ an incomplete pairwise comparison matrix. We then define $K_{i}$ as the number of the positive off-diagonal element $a_{i j}$ for $i=1, \ldots, n$.

As in the case of complete information, $w_{i}$ is the $i^{\text {th }}$ self-evaluation value and $\left(a_{i} w-w_{i}\right) / K_{i}$ is called the $i^{t h}$ non-self-evaluation value. This definition of non-self-evaluation value is a natural extension of the complete information case, because in this case we have $K_{i}=n-1$ for $i=1, \ldots, n$. Hence the definitions of the self-evaluation value and the non-self-evaluation value in AHP with incomplete information include those in AHP with complete information.

\section{Optimization Models for the Incomplete Information Case}

This section discusses the incomplete information case in the model-based AHP which is based on the self-evaluation and the non-self-evaluation. The complete information case is dealt as a special case of the incomplete information case. 
By introducing the generalized definitions of the self-evaluation value $w_{i}$ and the non-self evaluation value $\left(a_{i} w-w_{i}\right) / K_{i}$ into $P_{1}$ and. $P_{2}$, we formulate the following discrepancy minimization problems with the ratios of the self-evaluation value to the non-self-evaluation value:

$$
\begin{aligned}
& \left(Q_{1}\right) \quad \max _{w>0} \min \left\{\frac{a_{1} w-w_{1}}{K_{1} w_{1}}, \ldots, \frac{a_{n} w-w_{n}}{K_{n} w_{n}}\right\} \\
& \left(Q_{2}\right) \quad \min _{w>0} \max \left\{\frac{a_{1} w-w_{1}}{K_{1} w_{1}}, \ldots, \frac{a_{n} w-w_{n}}{K_{n} w_{n}}\right\}
\end{aligned}
$$

$P_{1} / P_{2}$ is identical to $Q_{1} / Q_{2}$ with the complete information, that is $K_{i}=n-1$ for $i=1, \ldots, n$. For the matrix $A$, we define the $i^{t h}$ row vector $\hat{a}_{i}=\left(a_{i}-e_{i}\right) / K_{i}$ for $i=1, \ldots, N$, where $e_{i}$ is the $i^{\text {th }}$ unit row vector. The matrix of $\hat{a}_{i}$ is then defined as:

$$
\hat{A}=\left[\begin{array}{c}
\hat{a}_{1} \\
\vdots \\
\hat{a}_{n}
\end{array}\right]
$$

Lemma 3 Suppose that an incomplete pairwise comparison matrix $A$ is irreducible. Then $\hat{A}$ is also nonnegative and irreducible.

Proof: Since $\hat{a}_{i i}=\left(a_{i i}-1\right) / K_{i}=0$ and $\hat{a}_{i j}=a_{i j} / K_{i}, \hat{A}$ is nonnegative and irreducible.

Lemma 4 Suppose that an incomplete pairuise comparison matrix $A$ is irreducible. Then the principal eigenvalue of $\hat{A}$ is a single root of its characteristic equation and there exists a positive principal eigenvector of $\hat{A}$.

Proof: It is directly followed from Lemma 3 and Theorem 1.

The following two theorems state the relationship between a principal eigenvector of $\hat{A}$ and an optimal solution of $Q_{1}$ or $Q_{2}$.

Theorem 5 Suppose that $A$ is nonnegative and irreducible. Let $v$ be any positive $n$-dimensional vector other than a principal eigenvector of $A$, then

$$
\min \left\{\frac{a_{1} v}{v_{1}}, \ldots, \frac{a_{n} v}{v_{n}}\right\}<\lambda_{\max }<\max \left\{\frac{a_{1} v}{v_{1}}, \ldots, \frac{a_{n} v}{v_{n}}\right\},
$$

where $\lambda_{\max }$ is the principal eigenvalue of $A$.

Proof: We denote the transpose operator for a matrix or a vector by $T$. Let $\lambda_{\max }$ be the principal eigenvalue of $A$. Then, the principal eigenvalue of $A^{T}$ is $\lambda_{\max }$ and there exists a positive principal vector $u$ of $A^{T}$ corresponding to $\lambda_{\max }$ since $A^{T}$ is also nonnegative and irreducible.

We will consider the two assumptions $a_{i} v / v_{i} \leq \lambda_{\max }$ for every $i=1, \ldots, n$ and $a_{i} v / v_{i} \geq \lambda_{\max }$ for every $i=1, \ldots, n$, and lead to contradiction under either assumption.

First suppose that $a_{i} v / v_{i} \leq \lambda_{\max }$ for every $i=1, \ldots, n$. Since $v$ is not a principal eigenvector of $A$, there exists an index $l$ such that $a_{l} v / v_{l}<\lambda_{\max }$. It follows from the positiveness of $v$ that

$$
A v \leq \lambda v \text { and } a_{l} v<\lambda v_{l} \text { for some } l .
$$

This means from $u^{T} A=\lambda_{\max } u^{T}$ that

$$
0>\sum_{i=1}^{n} u_{i}\left(a_{i} v-\lambda_{\max } v_{i}\right)=\sum_{i=1}^{n} u_{i} a_{i} v-\lambda_{\max } u_{i} v_{i}=\lambda_{\max } u^{T} v-\lambda_{\max } u^{T} v=0
$$

which is contradiction.

The other assumption also leads to contradiction in the same manner. 
Theorem 6 Suppose that an incomplete pairwise comparison matrix $A$ is irreducible. An optimal solution of $Q_{1}$ is equal to a positive principal eigenvector of $\hat{A}$, and vice versa. An optimal solution of $Q_{2}$ is also equal to a positive principal eigenvector of $\hat{A}$, and vice versa.

Proof: It follows from Lemma 3 that $\hat{A}$ is nonnegative and irreducible. Let $\hat{\lambda}_{\max }$ be the principal eigenvalue of $\hat{A}$. Then it follows from (2) of Theorem 2 that

$$
\max _{w>0} \min \left\{\frac{\hat{a}_{1} w}{w_{1}}, \ldots, \frac{\hat{a}_{n} w}{w_{n}}\right\}=\hat{\lambda}_{\max }=\min _{w>0} \max \left\{\frac{\hat{a}_{1} w}{w_{1}}, \ldots, \frac{\hat{a}_{n} w}{w_{n}}\right\} .
$$

Since $\hat{a}_{i} w=\left(a_{i}-e_{i}\right) w / K_{i}=\left(a_{i} w-w_{i}\right) / K_{i}$, the left hand and the right hand of (5) are equivalent to $Q_{1}$ and $Q_{2}$, respectively. Hence it follows from Theorem 2 that a positive principal eigenvector of $\hat{A}$ is an optimal solution of both $Q_{1}$ and $Q_{2}$.

Let $v$ be any positive vector other than a principal eigenvector of $\hat{A}$. Since $\hat{A}$ is nonnegative and irreducible, it follows from Theorem 5 that

$$
\begin{aligned}
\min \left\{\frac{\hat{a}_{1} v}{v_{1}}, \ldots, \frac{\hat{a}_{n} v}{v_{n}}\right\} & =\min \left\{\frac{a_{1} v-v_{1}}{K_{1} v_{1}}, \ldots, \frac{a_{n} v-v_{n}}{K_{n} v_{n}}\right\}<\hat{\lambda}_{\max } \\
& <\max \left\{\frac{\hat{a}_{1} v}{v_{1}}, \ldots, \frac{\hat{a}_{n} v}{v_{n}}\right\}=\max \left\{\frac{a_{1} v-v_{1}}{v_{1}}, \ldots, \frac{a_{n} v-v_{n}}{v_{n}}\right\} .
\end{aligned}
$$

Therefore $v$ is not an optimal solution of either $Q_{1}$ nor $Q_{2}$.

In order to combine the two optimization problems $Q_{1}$ and $Q_{2}$, we propose the following discrepancy minimization problem that evaluates the ratios of the self-evaluation value to non-self-evaluation value and their reciprocal:

$$
\left(Q_{3}\right) \quad \min _{w>0} \max \left\{\frac{a_{1} w-w_{1}}{K_{1} w_{1}}, \ldots, \frac{a_{n} w-w_{n}}{K_{n} w_{n}}, \frac{K_{1} w_{1}}{a_{1} w-w_{1}}, \ldots, \frac{K_{n} w_{n}}{a_{n} w-w_{n}}\right\}
$$

Lemma 7 Suppose that an incomplete pairwise comparison matrix $A$ is irreducible. Then $Q_{3}$ has an optimal solution.

Proof: Let $\hat{\lambda}_{\max }$ be the principal eigenvalue of $\hat{A}$. Then it follows from Theorem 2 that for every positive vector $v$

$$
\max \left\{\frac{a_{1} v-v_{1}}{K_{1} v_{1}}, \ldots, \frac{a_{n} v-v_{n}}{K_{n} v_{n}}, \frac{K_{1} v_{1}}{a_{1} v-v_{1}}, \ldots, \frac{K_{n} v_{n}}{a_{n} v-v_{n}}\right\} \geq \max \left\{\frac{a_{1} v-v_{1}}{K_{1} v_{1}}, \ldots, \frac{a_{n} v-v_{n}}{K_{n} v_{n}}\right\} \geq \hat{\lambda}_{\max }
$$

and

$$
\begin{aligned}
\max \left\{\frac{a_{1} v-v_{1}}{K_{1} v_{1}}, \ldots, \frac{a_{n} v-v_{n}}{K_{n} v_{n}}, \frac{K_{1} v_{1}}{a_{1} v-v_{1}}, \ldots, \frac{K_{n} v_{n}}{a_{n} v-v_{n}}\right\} & \geq \max \left\{\frac{K_{1} v_{1}}{a_{1} v-v_{1}}, \ldots, \frac{K_{n} v_{n}}{a_{n} v-v_{n}}\right\} \\
= & \left(\min \left\{\frac{a_{1} v-v_{1}}{K_{1} v_{1}}, \ldots, \frac{a_{n} v-v_{n}}{K_{n} v_{n}}\right\}\right)^{-1} \geq \frac{1}{\hat{\lambda}_{\max }} .
\end{aligned}
$$

Let $w$. be a positive principal eigenvector of $\hat{A}$. Then we have

$$
\begin{aligned}
& \max \left\{\frac{a_{1} w-w_{1}}{K_{1} w_{1}}, \ldots, \frac{a_{n} w-w_{n}}{K_{n} w_{n}}, \quad\right.\left.\frac{K_{1} w_{1}}{a_{1} w-w_{1}}, \ldots, \frac{K_{n} w_{n}}{a_{n} w-w_{n}}\right\}=\max \left\{\hat{\lambda}, \frac{1}{\hat{\lambda}_{\max }}\right\} \\
& \leq \max \left\{\frac{a_{1} v-v_{1}}{K_{1} v_{1}}, \ldots, \frac{a_{n} v-v_{n}}{K_{n} v_{n}}, \frac{K_{1} v_{1}}{a_{1} v-v_{1}}, \ldots, \frac{K_{n} v_{n}}{a_{n} v-v_{n}}\right\}
\end{aligned}
$$

for every positive vector $v$.

Theorem 8 Suppose that an incomplete pairwise comparison matrix $A$ is irreducible. Let $\hat{\lambda}_{\max }$ be the principal eigenvalue of $\hat{A}$, then the optimal values of $Q_{3}$ is $\max \left\{\hat{\lambda}_{\max }, \hat{\lambda}_{\max }^{-1}\right\}$. Furthermore an optimal solution of $Q_{3}$ is equal to a positive principal eigenvector of $\hat{A}$, and vice versa. 
Proof: The optimal value is already shown in the proof of Lemma 7. Therefore, we only have to show that an optimal solution of $Q_{3}$ is a positive principal eigenvector of $\hat{A}$.

Suppose that $v$ is any positive vector other than a positive principal eigenvector of $\hat{A}$. Then it follows from Theorem 5 that $\left(\min \left\{\left(a_{1} v-v_{1}\right) / K_{1} v_{1}, \ldots,\left(a_{n} v-v_{n}\right) / K_{n} v_{n}\right\}\right)^{-1}>\hat{\lambda}_{\max }^{-1}$ and that $\max \left\{\left(a_{1} v-v_{1}\right) / K_{1} v_{1}, \ldots,\left(a_{n} v-v_{n}\right) / K_{n} v_{n}\right\}>\hat{\lambda}_{\text {tmax }}$. This means that

$$
\max \left\{\frac{a_{1} v-v_{1}}{K_{1} v_{1}}, \ldots, \frac{a_{n} v-v_{n}}{\bar{K}_{n} v_{n}}, \frac{K_{1} v_{1}}{a_{1} v-v_{1}}, \ldots, \frac{K_{n} v_{n}}{a_{n} v-v_{n}}\right\}>\max \left\{\hat{\lambda}_{\max }, \frac{1}{\hat{\lambda}_{\max }}\right\} .
$$

Theorem 8 asserts that $Q_{1}, Q_{2}$ and $Q_{3}$ have the same optimal solutions.

\section{Some Properties of the Optimization Models}

In order to describe the structure of the incomplete pairwise comparisons for $n$ alternatives, we consider the following undirected graph with $n$ nodes: If a pair $(i, j)$ of alternatives $i$ and $j$ is compared by a decision maker, the arc $(i, j)$ between the node $i$ and the node $j$ is defined. We denote the graph corresponding to the incomplete pairwise comparison matrix $A$ by $G(A)$. In the case of the incomplete information, the graph is not complete.

Harker method is available for evaluating the weight vector from an irreducible incomplete pairwise matrix $A$ of order $n$ and the weight vector of Harker method is a principal eigenvector of $A$ with the diagonal entry $a_{i i}$ replaced by $n-K_{i}$. Therefore we formulate the following optimization problem corresponding to Harker method:

$$
\min _{w>0} \max \left\{\frac{a_{1} w}{w_{1}}+n-K_{1}-1, \ldots, \frac{a_{n} w}{w_{n}}+n-K_{n}-1\right\} .
$$

Lemma 9 Suppose that $A$ is an incomplete pairwise comparison matrix of order $n$, and that it is irreducible. An optimal solution of $Q_{4}$ is equal to a principal eigenvector of $A$ with the diagonal entry $a_{i i}$ replaced by $n-K_{i}$, and vice versa.

The proof is omitted.

Theorem 10 Suppose that $A$ is an incomplete pairwise comparison matrix of order $n$, and that it is irreducible. Assume that $K_{1}=\cdots=K_{n}$. An optimal solution of $Q_{1}, Q_{2}$ and $Q_{3}$ is equal to an optimal solution of $Q_{4}$, and vice versa.

Proof: From theorems 6 and 8, the optimal solutions of $Q_{1}, Q_{2}$ and $Q_{3}$ are equivalent, we only have to show one of them is equivalent to $Q_{4}$.

If $K_{1}=\cdots=K_{n}$, then it follows that

$$
\begin{aligned}
\min _{w>0} \max \left\{\frac{a_{1} w-w_{1}}{K_{1} w_{1}}, \ldots, \frac{a_{n} w-w_{n}}{K_{n} w_{n}}\right\} & =\frac{1}{K_{1}} \min _{w>0} \max \left\{\frac{a_{1} w-w_{1}}{w_{1}}, \ldots, \frac{a_{n} w-w_{n}}{w_{n}}\right\} \\
& =\frac{1}{K_{1}} \min _{w>0} \max \left\{\frac{a_{1} w}{w_{1}}-1, \ldots, \frac{a_{n} w}{w_{n}}-1\right\} \\
& =\frac{1}{K_{1}} \min _{w>0} \max \left\{\frac{a_{1} w}{w_{1}}, \ldots, \frac{a_{n} w}{w_{n}}\right\}-\frac{1}{K_{1}} .
\end{aligned}
$$

Hence an optimal solution of $Q_{2}$ is equal to an optimal solution of $\min _{w>0} \max \left\{a_{1} w / w_{1}, \ldots, a_{n} w / w_{n}\right\}$. Furthermore since $K_{1}=\cdots=K_{n}, Q_{4}$ is equivalent to

$$
\min _{w>0} \max \left\{\frac{a_{1} w}{w_{1}}, \ldots, \frac{a_{n} w}{w_{n}}\right\}+n-K_{1}-1 .
$$

Therefore an optimal solution of $Q_{4}$ is equal to $\min _{w>0} \max \left\{a_{1} w / w_{1}, \ldots, a_{n} w / w_{n}\right\}$.

All nodes of the graph $G(A)$ have the same degree if and only if $K_{1}=\cdots=K_{n}$. Such a graph is called regular. The above theorem can be also expressed in terms of graphs: 
Corollary 11 Suppose that $A$ is an incomplete pairwise comparison matrix of order $n$, and that $G(A)$ is connected and regular. An optimal solution $Q_{4}$ is equal to an optimal solution of $Q_{1}, Q_{2}$ and $Q_{3}$, respectively, and vice versa.

The following theorem guarantees that both $Q_{3}$ and $Q_{4}$ provide non-biased weights for the consistent pairwise comparison values.

Theorem 12 Suppose that $A$ is an incomplete pairwise comparison matrix of order $n$, and that it is irreducible. Assume that the optimal value of $Q_{4}$ is $n$. An optimal solution of $Q_{4}$ is equal to an optimal solution of $Q_{3}$.

Proof: Suppose that $w$ is any optimal solution of $Q_{4}$. Since $A$ with all diagonal entries $a_{i i}$ replaced by $n-K_{i}$ is a nonnegative irreducible matrix and the optimal value of $Q_{4}$ is $n$, it follows from Theorem 2 that $n=a_{1} w / w_{1}+n-K_{1}-1=\cdots=a_{n}^{\prime} w / w_{n}+n-K_{n}-1$. Therefore we have $\left(a_{i} w-w_{i}\right) / w_{i}=K_{i}$ for every $i=1, \ldots, n$. This means form Theorem 8 that

$$
\begin{aligned}
\max \left\{\hat{\lambda}_{\max }, \frac{1}{\hat{\lambda}_{\max }}\right\} & \leq \max \left\{\frac{a_{1} w-w_{1}}{K_{1} w_{1}}, \ldots, \frac{a_{n} w-w_{n}}{K_{n} w_{n}}, \frac{K_{1} w_{1}}{a_{1} w-w_{1}}, \ldots, \frac{K_{n} w_{n}}{a_{n} w-w_{n}}\right\} \\
& =1 \leq \max \left\{\hat{\lambda}_{\max }, \frac{1}{\hat{\lambda}_{\max }}\right\},
\end{aligned}
$$

where $\hat{\lambda}_{\max }$ is the principal eigenvalue of $\hat{A}$. Therefore $w$ is an optimal solution of $Q_{3}$.

Corollary 13 Suppose that $A$ is an incomplete pairwise comparison matrix of order $n$, and that it is irreducible. Assume that the optimal value of $Q_{3}$ is 1 . An optimal solution of $Q_{3}$ is equal to an optimal solution of $Q_{4}$.

Proof: It is trivial from the proof of Theorem 12.

The above two assertions means from Theorem 8 that an optimal solution of $Q_{4}$ is an optimal solution of $Q_{i}$ for $i=1,2,3$, respectively.

Corollary 14 Suppose that $A$ is an incomplete pairwise comparison matrix of order $n$, and that it is irreducible. Assume that the optimal value of $Q_{4}$ is $n$. An optimal solution of $Q_{4}$ is equal to an optimal solution of $Q_{i}$ for $i=1,2,3$, respectively.

Here, we consider the special structure of $G(A)$, a spanning tree.

Corollary 15 Suppose that $A$ is an incomplete pairwise comparison matrix of order $n$, and that $G(A)$ is a spanning tree. An optimal solution of $Q_{4}$ is equal to an optimal solution of $Q_{i}$ for $i=1,2,3$, respectively.

Proof: If $G(A)$ is a spanning tree, the rank of the node-arc incidence matrix of $G(A)$ is $n-1$. Therefore there exists a positive vector $w=\left(w_{1}, \ldots, w_{n}\right)^{T}$ such that $K_{i} \hat{a}_{i j}=w_{i} / w_{j}$ for $\hat{a}_{i j}>0$ and we have

$$
\frac{\hat{a}_{i} w}{w_{i}}=\frac{a_{i} w-w_{i}}{K_{i} w_{i}}=\frac{\sum_{a_{i j}>0} w_{i}-w_{i}}{K_{i} w_{i}}=\frac{\left(K_{i}+1\right) w_{i}-w_{i}}{K_{i} w_{i}}=1
$$

for every $i=1, \ldots, n$. Hence the optimal value of $Q_{3}$ is 1 , which implies by Corollary 13 that the optimal value of $Q_{4}$ is $n$. This assertion is held by Corollary 14 .

\section{References}

Furuya, S. (1957) Matrix and Determinant, (in Japanese) Tokyo: Baifu-kan.

Harker, P.T. (1987), "Alternative modes of questioning in the analytic hierarchy process," Mathematical Modelling, 9, 353-360.

Sekitani, K and Yamaki, N. (1999), "A logical interpretation for the eigenvalue method in AHP", Journal of the Operations Research Society of Japan, In printing.

Takahashi, I. (1999) "Recent theoretical developments of AHP and ANP in Japan". Proceedings of ISAHP Kobe.

Takayama, A. (1985) Mathematical Economics, Cambridge:Cambridge University Press. 